\title{
Cefotiam Hexetil Hydrochloride
}

National Cancer Institute

\section{Source}

National Cancer Institute. Cefotiam Hexetil Hydrochloride. NCI Thesaurus. Code C98226.

The hydrochloride salt of cefotiam hexetil, an ester prodrug of cefotiam for parenteral use. Cefotiam is a semi-synthetic, beta-lactam cephalosporin antibiotic with antibacterial activity. After administration of cefotiam hexetil hydrochloride the ester bond is cleaved, releasing active cefotiam. 